\title{
Ethnologies
}

\section{Mise en tourisme des produits du terroir, événements festifs et mutations du patrimoine ethnologique en Provence (France)}

\section{Laurent Sébastien Fournier}

Volume 32, numéro 2, 2010

Tourisme culturel

Cultural Tourism

URI : https://id.erudit.org/iderudit/1006307ar

DOI : https://doi.org/10.7202/1006307ar

Aller au sommaire du numéro

Éditeur(s)

Association Canadienne d'Ethnologie et de Folklore

ISSN

1481-5974 (imprimé)

1708-0401 (numérique)

Découvrir la revue

Citer cet article

Fournier, L. S. (2010). Mise en tourisme des produits du terroir, événements festifs et mutations du patrimoine ethnologique en Provence (France).

Ethnologies, 32(2), 103-144. https://doi.org/10.7202/1006307ar
Résumé de l'article

Cet article s'intéresse au passage de la reconnaissance d'un patrimoine ethnologique à sa mise en tourisme. L'exemple de l'oléiculture est privilégié ici en raison de l'engouement contemporain mondial pour les produits liés à l'olivier ( Olea europaea l.). Des événements festifs mettant en scène les produits oléicoles à l'intention de nouveaux publics essentiellement touristiques et urbains sont d'abord observés et décrits. Il s'agit ensuite d'étudier les processus de requalification qui accompagnent le passage de la mise en patrimoine à la mise en tourisme, sur un plan à la fois spatial, social, symbolique et sensoriel. 


\section{Mise EN TOURISME DES PRODUITS DU TERROIR, ÉVÉNEMENTS FESTIFS ET MUTATIONS DU PATRIMOINE ethnologiQue en Provence (France)}

\section{Laurent Sébastien Fournier \\ Université de Nantes}

Afin de mieux comprendre les modalités de construction des cultures touristiques et les réflexes développés face au tourisme par les sociétés d'accueil, le présent travail consiste à essayer de mesurer les conséquences sur le tourisme des opérations récurrentes de mise en valeur du patrimoine $e^{1}$ que connaît notre époque, en examinant un ensemble de problèmes concrets qui se posent en aval des opérations de patrimonialisation d'éléments du patrimoine ethnologique. Notre travail est basé sur l'exemple de l'oléiculture et des produits liés à l'olivier (Olea europaea l.), en raison de l'engouement que ces derniers suscitent dans un contexte où les questions liées à l'environnement, au patrimoine rural et à la qualité de l'alimentation gagnent en importance.

La revitalisation et la patrimonialisation récentes de l'olivier et des produits oléicoles sont des phénomènes qui s'inscrivent dans un vaste ensemble de pratiques de relance culturelle et de redécouverte des traditions. A la différence d'un grand nombre d'opérations culturelles, centrées sur une dimension locale, la vocation de l'olivier est le plus souvent considérée comme universelle : l'olivier est un arbre et à ce titre fait partie de la nature, il est chargé de symboles, et les produits qui en dérivent sont mondialement consommés. Dès lors, la question n'est pas tant de renouveler le constat de la patrimonialisation de l'oléiculture par un ensemble d'acteurs locaux, que d'en cerner précisément les conséquences et les effets multiples, sur un plan plus

1. Malgré la distinction opérée par Davallon (1991 : 97), nous utiliserons le plus souvent comme synonymes les expressions « mise en valeur» et « valorisation ». Par ailleurs, nous accorderons un sens identique aux expressions « mise en patrimoine » et «patrimonialisation». 
général. De même, il ne s'agit pas de nous situer par rapport aux débats qui opposent, au sein des études consacrées au tourisme, les partisans du point de vue individuel et ceux du point de vue collectif, ou les psychosociologues et les socio-économistes, mais plutôt de comprendre comment s'articulent et interagissent mise en patrimoine et mise en tourisme. A un premier niveau, notre problématique est la suivante: quelles sont les conséquences culturelles, sociales, économiques de la valorisation patrimoniale de l'oléiculture? A un second niveau, il s'agira de montrer que la patrimonialisation est largement déterminée par l'ouverture au tourisme des sociétés considérées, en l'occurrence celles du pourtour méditerranéen.

Dans un premier temps, selon une logique inductive généralement admise au sein des sciences sociales, nous présenterons un ensemble de faits et de données empiriques concernant des événements festifs ou culturels qui mettent en scène les produits oléicoles à l'intention de nouveaux publics essentiellement touristiques et urbains, c'est-à-dire extérieurs au monde d'origine local et rural de ces produits. La comparaison des processus de valorisation à l'échelle d'un territoire, la vallée des Baux-de-Provence, et à l'échelle d'un équipement culturel prestigieux, la Chartreuse de Villeneuve-lès-Avignon, Centre national des écritures du spectacle, témoignera du mouvement de fond qui a conduit les produits oléicoles du statut de production agricole traditionnelle à celui de "symbole alimentaire mondialisé $»^{2}$, et qui les a rendus disponibles pour les touristes quelle que soit leur origine.

L'exposé des faits conduira, dans une perspective plus analytique ou théorique, à une discussion sur les dynamiques à l'œuvre dans les processus de patrimonialisation en contexte touristique. En critiquant l'hypothèse de la "muséalisation » ou de la «pétrification patrimoniale » (Jeudy 1999: 171), selon laquelle le patrimoine serait pour l'essentiel une construction artificielle, et en privilégiant une approche en termes d'économie symbolique (Godelier 1996; La Pradelle 1996) pour comprendre quelle est la nature de la relation qui unit patrimoine et valeur, nous serons amenés à insister sur la notion de requalification. Dynamique, cette notion sera considérée dans sa triple acception spatiale (Micoud 1991 ; Davallon 1991), sociale et symbolique (Bromberger et Chevallier 1999; Rautenberg et al. 2000),

2. Voir le rapport d'enquêtes et de collectes (non publié) intitulé L'huile d'olive : de la culture traditionnelle au symbole alimentaire mondialisé (2002). 
et sensorielle (Albert 1989 ; Candau 2000). Elle constitue un outil heuristique important pour comprendre ce qui advient lorsque l'image et le sens d'un produit ou d'un groupe de produits sont confrontés au changement social. Le tourisme, en important des références nouvelles, modifie le sens des références existantes et déplace le centre de gravité sémantique des objets culturels. C'est ainsi que l'univers de référence des produits oléicoles passe en Provence de l'agriculture à l'art et à la haute culture, du rural à l'urbain, du populaire à l'élite, du vivrier au gastronomique, du travail des champs au loisir cultivé, du local au global. De telles mutations éclairent les recompositions inévitables des sociétés réceptrices de touristes et leurs systèmes de références culturelles. Elles montrent comment le patrimoine ethnologique rejoint la mise en tourisme et sert de lieu de médiation entre les identités locales et les regards extérieurs des touristes qui de plus en plus les traversent et inévitablement les bouleversent.

\section{Etudes de cas : événements festifs, produits du terroir et mise en tourisme de la culture locale}

Ce travail vise en premier lieu à rendre compte d'une série d'enquêtes ethnologiques menées en Provence, dans le département des Bouchesdu-Rhône et dans le Gard, depuis 2002. Ces enquêtes, qui se sont concentrées sur des opérations de patrimonialisation liées aux produits oléicoles, interrogent aussi bien le champ du tourisme et du patrimoine culturel que celui des études de la communication des institutions culturelles. Elles montrent ainsi comment la mise en patrimoine se conjugue avec la mise en tourisme et conduit à modifier le regard porté sur certains éléments clés de l'environnement local.

Le premier terrain de recherche correspond à un territoire, celui de la vallée des Baux-de-Provence (Bouches-du-Rhône). La valorisation patrimoniale et touristique de l'oléiculture y est engagée depuis plus de trente ans, notamment autour de la création de fêtes liées aux produits oléicoles et grâce à l'action des filières professionnelles qui ont progressivement mis en œuvre des stratégies pour obtenir un certain nombre de labels de qualité. Plus récemment, le caractère remarquable de l'oléiculture a servi de prétexte à une collecte muséographique menée dans le cadre de la préfiguration du Musée des civilisations de l'Europe et de la Méditerranée (antenne marseillaise du Musée national des Arts 
et Traditions populaires), qui s'inscrit elle-même dans l'ensemble des procédés de valorisation développés sur ce territoire.

Une deuxième étude de cas concerne les Oléades, un événement conçu en 2000 par la Chartreuse de Villeneuve-lès-Avignon, Centre national des écritures du spectacle, monument historique labellisé "Centre culturel de rencontre » par le ministère de la Culture. Autour d'expositions et d'animations dont nous analyserons le contenu, une structure totalement extérieure au monde des professionnels de l'olivier a voulu mettre en place ici une opération de mise en valeur patrimoniale qui s'adresse à un large public dans une optique avérée de développement touristique.

\section{La valorisation patrimoniale de l'oléiculture dans la vallée des Baux}

Dans la vallée des Baux, les enquêtes ethnologiques ont permis une analyse précise des relances festives liées à l'oléiculture, à partir de laquelle des étapes de valorisation patrimoniale et culturelle peuvent être décrites. Dès le XIXe siècle, l'écrivain et folkloriste Frédéric Mistral fait de l'olivier un symbole provençal, à travers son œuvre poétique, lexicographique, journalistique et muséographique. Le travail de l'olivier et sa place dans la culture régionale sont intégrés aux collections du Muséon arlaten, le musée d'ethnographie provençale d'Arles, dès son ouverture en 1896. Un peintre comme Van Gogh, lorsqu'il séjourne en Provence, s'intéresse à la lumière particulière que l'olivier condense. Après la Libération, en 1947, une Confrérie des oliverons, aux Bauxde-Provence, rassemble félibres, artistes, et notables. Des gravures y sont réalisées par le typographe Louis Jou, qui travaille avec Fernand Benoît et Pierre-Louis Duchartre ${ }^{3}$. En 1956, le gel massif des oliviers provençaux inspire des textes et de nouvelles actions. En particulier, un concours de sculptures réalisées par des artistes dans les troncs des arbres gelés est organisé, dans le champ d'intérêt du ministère de l'Agriculture. Mais c'est surtout à partir des années 1970 que l'oléiculture est relancée, revitalisée, dans un cadre local mais aussi européen : une série d'initiatives très diverses voit le jour, combinant programmes économiques, projets touristiques et culturels.

3. Respectivement rattachés au Muséon arlaten et au Musée national des Arts et Traditions populaires. 
Depuis les années 1980 et la remise en production des arbres recépés après le gel de 1956, la valorisation patrimoniale de l'oléiculture passe chez les professionnels par la recherche de labels de qualité et pour l'ensemble des acteurs locaux par des projets thématiques d'animation ou de développement touristique et culturel. Dans ce contexte sont créées des fêtes thématiques locales consacrées à l'oléiculture, qui n'avaient pas d'équivalent dans la société agro-pastorale traditionnelle. Selon plusieurs interlocuteurs rencontrés dans le village de Mouriès, où sont organisées la fête des olives vertes (célébrée en septembre depuis 1968) et la fête de l'huile nouvelle (célébrée en décembre depuis 1991), ces événements sont plus un prétexte pour faire vivre le village que pour faire vivre l'olive, qui n'a pas besoin de ces fêtes. De manière significative, dans l'organigramme de la municipalité de Mouriès, le comité des fêtes qui a créé ces événements qui contribuent à la mise en valeur patrimoniale de l'oléiculture se confond avec l'office du tourisme local. Le ressort de ces activités de mise en valeur, ainsi, serait plus symbolique qu'économique. L'olive fêtée tiendrait son statut patrimonial de son rôle dans la construction de l'identité locale plus que de son rendement économique. Elle aurait un impact médiatique propre à attirer les touristes, et pas seulement un statut de produit agricole. A travers les fêtes qui la mettent en patrimoine, l'olive ne serait donc pas seulement une ressource agricole, mais induirait aussi un développement de l'économie touristique locale.

Ce type de discours a l'avantage de placer les phénomènes de patrimonialisation dans la logique du symbolique, et cautionne l'idée selon laquelle l'olive ne vaut qu'en raison de sa place emblématique parmi les symboles de l'identité provençale. A ce compte, l'olive est patrimoniale parce qu'elle représente l'identité locale, elle se reflète dans un ensemble de discours qui ont construit cette identité, elle est un indice de choix pour aller à sa découverte, et elle est bonne à consommer par les touristes. Et de fait, les utilisations des produits oléicoles dans les fêtes sont très variées et s'adressent à des publics hétérogènes, depuis la consommation entre gens du village du traditionnel aïoli (le repas populaire de la fête votive) jusqu'au concours dérisoire de cassage d'olive, aux visites d'oliveraies et de moulins ou aux foires-expositions de produits du terroir dirigées vers un public extra-local et principalement touristique (Fournier 2005).

Malgré ce lien privilégié avec la construction de l'identité locale et sa médiatisation devant un public extérieur, bien mis en évidence par 
la mention récurrente des fêtes de l'olive dans les agendas culturels édités par le comité départemental du tourisme des Bouches-du-Rhône, les aspects économiques ne sont pas absents du dossier. Les projets d'animation et de développement culturel sont relayés par les préoccupations des acteurs des filières professionnelles. Mouliniers, confiseurs et oléiculteurs sont rassemblés autour du syndicat interprofessionnel oléicole de la vallée des Baux, qui se bat pour l'obtention de labels de qualité permettant une production rentable.

Cette présence des acteurs économiques dans la définition du patrimoine n'est sans doute pas étrangère à la diversification des formes culturelles et des représentations liées à l'oléiculture. Par exemple, l'obtention dans les Alpilles du label «Site remarquable du goût $»^{4}$ a contribué à rapprocher l'oléiculture de la gastronomie ; les labels «Paysage de reconquête ${ }^{5}$ et "Route de l'olivier ${ }^{6}$ ont révélé le rôle des oliviers dans la construction de sites patrimoniaux et d'itinéraires culturels ; l'obtention du label "Appellation d'origine contrôlée » ${ }^{7}$ a permis enfin de rapprocher les univers de référence de l'oléiculture et de l'œnologie.

Les produits oléicoles forment ainsi un champ complexe régi par des forces de nature très différente. C'est sans doute ce qui explique que les fêtes consacrées à ces produits, à la différence des fêtes anciennes dont le programme était reconduit immuablement chaque année, sont des constructions mouvantes qui s'étoffent avec des éléments nouveaux. Ainsi, au fur et à mesure que l'oléiculture gagne en légitimité patrimoniale, le programme des fêtes se diversifie en intégrant des activités qui n'ont plus rien à voir avec l'oléiculture (défilé de voitures anciennes, concours de chiens, etc.), et parallèlement, l'oléiculture diversifie ses moyens de mise en valeur et se fait représenter comme patrimoine à travers des actions de plus en plus nombreuses.

4. Label du Conseil national des arts culinaires (1994).

5. Label du ministère de l'Environnement (1993).

6. Label créé par le Centre économique agricole de l'olivier avec l'appui de la C.E.E. (DG VI, Développement rural).

7. En 1997, la vallée des Baux obtient cinq labels A.O.C. : vin rosé, vin rouge, huile d'olive, olives vertes "cassées » et olives noires «piquées ». 
L'obtention de labels de qualité est un de ces moyens, mais il en existe d'autres, très souvent liés entre eux, et parmi lesquels nous citerons pêle-mêle la création d'une Confrérie des mouliniers, qui intronise annuellement des membres influents de la société locale sur le modèle des confréries vineuses, le rachat par la municipalité de Mouriès d'un moulin abandonné pour abriter l'office du tourisme local et héberger un centre culturel et un centre d'affaires, le projet d'un écomusée consacré à l'oléiculture, les réflexions relatives à la place du thème de l'oléiculture dans la mise en place de la charte du futur parc naturel régional des Alpilles, ou encore la malle pédagogique conçue par une association locale de sauvegarde du patrimoine et présentée aux touristes de passage lors des fêtes.

Ainsi, il existe un vaste ensemble de procédés de valorisation patrimoniale qui ouvre l'oléiculture au domaine du tourisme et qui témoigne de la variété des enjeux convoqués ainsi que des possibilités nombreuses de transposition à d'autres pratiques culturelles. Pour démontrer l'impact touristique et les conséquences de cette longue valorisation patrimoniale de l'oléiculture, l'exemple d'une collecte muséographique est très significatif.

\section{Collectes muséographiques et ouverture au tourisme}

Une campagne d'acquisition internationale lancée par la Réunion des musées nationaux en 2002 a été dirigée par deux conservateurs au Musée national des Arts et Traditions populaires, Jean-François Charnier et Liliane Kleber ${ }^{8}$. Le centre de cette opération de collecte, lancée simultanément en Provence, en Grèce, en Tunisie et au Maroc, se trouve en Provence, sur le territoire de la vallée des Baux, en raison notamment de l'obtention des labels A.O.C., de la présence de la commune oléicole la plus importante de France en termes de surface plantée (Mouriès), de l'implantation de la coopérative la plus importante de France (Maussane) et de la diversité des structures et des initiatives locales. La zone de la vallée des Baux est définie comme "un échantillon relativement représentatif de la situation de l'oléiculture sur le paysage français $»^{9}$.

8. Voir le rapport d'enquêtes et de collectes (non publié) intitulé L'huile d'olive : de la culture traditionnelle au symbole alimentaire mondialisé (2002).

9. Id. 
La problématique générale des enquêtes vise à rendre compte du passage, dans un contexte d'engouement généralisé pour les produits oléicoles et les oliviers, d'une économie agricole traditionnelle à une nouvelle économie marchande fondée sur le tourisme, avec tout ce que ces transformations impliquent de représentations nouvelles et d'innovations techniques. Les sites de production sont étudiés en tant qu' " unités écologiques », tandis que les consommateurs sont abordés par des enquêtes sous la forme de "portraits». Des acquisitions sont prévues concernant les systèmes techniques liés à la production, les témoins significatifs du contexte social et culturel et des implications symboliques de la pratique (biens domestiques liés à l'olive, représentations artistiques, etc.), le matériel utilisé pour la consommation et la promotion des huiles.

Cet exemple de campagne muséale d'acquisitions, basée sur une grille classique propre à rendre compte de la circulation sociale du produit depuis sa production jusqu'à sa consommation, nous est particulièrement précieux pour juger des conséquences de la mise en patrimoine de l'oléiculture. Ici, les muséographes interviennent après la phase de mise en patrimoine. Que vont-ils retenir de ce qui a été mis en valeur sur le terrain ? Comment vont-ils à leur tour transformer le sens des pratiques en choisissant de mettre en avant tel ou tel aspect? Une analyse sommaire du contenu de leur travail permet d'acquérir une meilleure connaissance des conséquences et des résultats de la patrimonialisation, d'envisager comment les muséographes proposent une image spécifique de l'oléiculture à un public extérieur, et finalement de comprendre le lien entre mise en patrimoine et mise en tourisme.

La base principale de la recherche, qui avait pour but de sélectionner des objets représentatifs pour l'exposition de référence du futur Musée des civilisations de l'Europe et de la Méditerranée à Marseille, a consisté en des enquêtes de terrain et des entretiens. Pour la zone de la vallée des Baux, des enquêtes ont été menées de manière à rencontrer les principaux acteurs locaux impliqués dans la valorisation et le développement de l'oléiculture. Des mouliniers, des oléiculteurs, des propriétaires de grandes exploitations, des responsables municipaux, des dégustateurs ont fourni des données sur les différentes orientations de la production oléicole. Ils ont témoigné de la complémentarité des acteurs locaux et néo-locaux dans la construction patrimoniale de l'oléiculture et ont souligné par exemple les spécificités de la culture biologique, de la recherche de qualité ou encore de la passion qui les 
lie à l'olivier. A travers ces discours ont été évoqués aussi les types de producteurs, les moyens de conditionnement des produits, les transformations liées à la mise en œuvre de l'A.O.C., les stratégies commerciales, la réception du produit, les rôles écologiques de l'olivier, les techniques culturales, les étapes de l'extraction de l'huile, les choix techniques liés à la production ou à la commercialisation des produits pour les touristes de passage.

Les discours collectés forment une trame sémantique qui influence la collecte d'objets, et qui correspond plus ou moins à la problématique générale qui a elle-même influencé les entretiens. La patrimonialisation, à ce compte, est une affaire de réglage des attentes et des désirs mutuels des acteurs locaux, des muséographes et des visiteurs extérieurs qu'elle implique. Parmi les objets acquis en Provence pour le musée, on compte principalement des objets fabriqués dans des matériaux modernes (plastique, métal), ce qui contraste avec les acquisitions faites en Tunisie et au Maroc, où les matériaux anciens (terre cuite, corne) continuent d'être utilisés. L'accent est porté sur les opérations techniques et les différents types de contenants, selon une approche qui rappelle la technologie culturelle d'André Leroi-Gourhan, et les acquisitions privilégient des objets du quotidien, comme le prévoient les normes habituelles de collecte dans le champ des arts et traditions populaires.

Le bilan que proposent les muséographes de cette première série d'enquêtes permet de clarifier le contexte historique, depuis le gel de 1956, de la relance patrimoniale de l'oléiculture ${ }^{10}$. Il permet de mettre en relation le développement de l'oléiculture et celui du tourisme, même s'il montre que l'intérêt économique réside davantage dans la hausse fantastique du prix du litre d'huile que dans un éventuel développement économique régional. La valeur du produit, ainsi, est davantage symbolique que réellement économique, dans la mesure où très peu de personnes vivent de l'oléiculture. Cependant, cette valeur symbolique est importante car elle peut être directement mise à disposition de ceux qui, comme les touristes, découvrent la région et cherchent à s'en approprier les symboles. De ce point de vue, la mise en tourisme peut se résumer par la mise à disposition des touristes d'éléments emblématiques du patrimoine local.

10. Voir le rapport L'huile d'olive [...] (2002). 


\section{Les étapes successives de la patrimonialisation}

L'observation des opérations successives qui, sur un territoire donné, consacrent la patrimonialisation de produits du terroir comme l'huile d'olive et l'olive de table, conduit à s'interroger sur les conséquences de toute opération de mise en patrimoine: comment la patrimonialisation permet-elle d'accroître la valeur d'une pratique sociale donnée? Qu'est-ce qui se trouve requalifié dans cette opération ? Qu'est-ce qui est exposé ? Dans quel but et pour quels destinataires?

Dans le cas que nous venons de décrire, plusieurs phases de patrimonialisation sont étroitement imbriquées et s'appuient les unes sur les autres pour former une chaîne de requalifications successives. Ici, il semble que la première phase de patrimonialisation en entraîne d'autres: de proche en proche, des objets de plus en plus banals et quotidiens sont en mesure d'être requalifiés. A cet effet, les objets collectés sont documentés par des entretiens qui révèlent leurs potentialités sociales et symboliques et permettent de leur reconnaître, parce qu'ils deviennent objets de discours, une valeur d'échange symbolique.

En considérant diachroniquement les différentes étapes de la mise en patrimoine de l'oléiculture, il est frappant de remarquer qu'une première patrimonialisation a d'abord eu lieu sous la forme d'une relance $^{11}$. Cette relance a permis, depuis une trentaine d'années, le développement de formes variées d'exposition du patrimoine oléicole. Ce dernier a servi de prétexte à des visites guidées, à des fêtes, à des jeux et à des cérémonies, d'emblème à des groupes folkloriques, d'enjeu à des concours. Il a été représenté lors de foires et d'expositions-ventes. Ces utilisations successives ont associé le patrimoine oléicole à une certaine image - localiste et traditionnelle - de l'identité provençale. Les outils traditionnels en bois et en vannerie (chevalets, scourtins),

11. Sur les caractéristiques des phénomènes de relance, voir Dupré (2000 : 398 401). Elle en distingue trois traits récurrents : s'appuyer sur des réseaux d'acteurs dont la stabilité et l'efficacité dépend parfois du succès de la relance ellemême ; réorganiser les différentes phases de la filière pour s'émanciper de certaines contraintes; ouvrir un registre de démonstration et de revendication propre à toucher un public élargi sur la base de références domestiques, civiques ou liées à l'opinion. Elle la conçoit comme une riposte à différentes dominations (d'ordre socio-technique, économique ou classificatoire) et estime qu'elle correspond au refus d'une identité prescrite. 
ainsi que l'image d'une industrie basée sur des savoir-faire ancestraux, ont été privilégiés au détriment des innovations et des évolutions réelles que connaissait le secteur de production des produits oléicoles.

Maintenant que de nouveaux opérateurs veulent mettre l'oléiculture au musée, ils entrent en concurrence avec les opérateurs de la première phase de patrimonialisation et doivent trouver le moyen de s'en distancier. Après que la première phase a requalifié les outils traditionnels tombés en désuétude, la seconde phase va alors se saisir des objets qui ont accompagné la première phase mais que cette dernière avait ignorés. Cette fois, ce sont des objets banals, quotidiens, utilitaires, faits de matériaux peu valorisés, qui vont être requalifiés à leur tour. Aussi, un facteur important de la patrimonialisation reste l'éloignement historique, qui permet seul de prendre conscience des sélections implicites auxquelles les acteurs de la valorisation procèdent et de remodeler ces sélections par la production d'un discours nouveau.

La patrimonialisation fonctionne grâce à la mise en place d'une interaction entre les objets et les discours qui les prennent en charge, entre les choses et les mots. Ce qui est dès lors au centre des logiques de mise en patrimoine, c'est la relation avec le monde d'origine des objets patrimonialisés. Cette relation est fonction de ce que l'opérateur patrimonial connaît de ce monde d'origine et de ce qu'il en entend. Ainsi, il ne peut y avoir d'origine que relative: les opérateurs de la première phase se sont situés par rapport aux techniques traditionnelles disparues; les muséographes de la collecte que nous avons évoquée se sont situés par rapport au monde déjà patrimonialisé qu'ils ont trouvé sur le terrain.

Dans ces conditions, il est intéressant d'observer la manière dont la patrimonialisation de l'oléiculture est recyclée par le musée de société. Le champ de réflexion des arts et traditions populaires, organisé autour d'une intégration de l'altérité esthétique figurée par une tension constitutive entre «art» et «populaire», prend pour univers de référence une oléiculture déjà patrimonialisée et axe surtout son discours sur la réception du label A.O.C. et sur les innovations techniques qui y sont liées. Cependant, il est à craindre que pour la plupart des visiteurs, les objets collectés ne dépassent pas la valeur documentaire, dans la mesure où ils sont directement issus du monde contemporain et où ils renvoient à un ensemble de constructions discursives sans lesquelles ils ne sont rien. Selon nous, la patrimonialité et la représentativité de ces 
objets n'est alors perceptible que par des acteurs extérieurs à la première phase de patrimonialisation, en particulier les touristes. A l'inverse, les acteurs de la première phase sont bien souvent critiques face à ces nouveaux objets. Dans la campagne d'acquisition que nous avons présentée, le travail de requalification s'effectue à partir d'un univers de référence déjà patrimonialisé dans une phase antérieure. Cette situation nous invite à tenter une comparaison avec d'autres situations où l'oléiculture est mise en patrimoine, pour parvenir à une meilleure compréhension des modalités mêmes de réception touristique de la mise en patrimoine.

\section{Les Oléades: le patrimoine oléicole exposé aux touristes}

Les Oléades sont un événement qui a été lancé en 2000 et qui a été organisé durant quelques années, de part et d'autre du Rhône, sur le site classé de la Chartreuse de Villeneuve-lès-Avignon et au Palais des Papes d'Avignon. Le contenu de cette manifestation, et notamment celui des expositions qui la composent, montre qu'il s'agit ici d'une structure extérieure au monde des professionnels de l'olivier qui met en place une opération de mise en valeur patrimoniale à l'intention d'un large public, principalement touristique.

Manifestation annuelle créée conjointement en 2000 par la Chartreuse de Villeneuve-lès-Avignon, le Centre national des écritures du spectacle, le Centre culturel de rencontres, et la Société d'économie mixte RMG, une société qui assure la gestion du Palais des Papes d'Avignon et qui tient à ce titre un rôle moteur dans l'économie touristique locale, les Oléades sont soutenues principalement par Monum, Centre des monuments nationaux, et par des organismes professionnels du monde de l'olivier. Le programme officiel de l'édition 2003, par exemple, nous renseigne sur le contenu général de cet événement organisé à la mi-février et qui rassemble sur un week-end un ensemble d'activités diversifiées. Des conférences sur des aspects thérapeutiques, historiques, symboliques, ou plus simplement techniques de l'oléiculture sont organisées. La première année, l'événement avait été lancé par un colloque consacré à l'oléiculture (Les Oléades 2001). Chaque année, des professionnels proposent une initiation à la taille des oliviers, des visites guidées thématiques sont mises en place, une exposition-vente de produits du terroir investit le Palais des Papes, des dégustations gastronomiques et des démonstrations culinaires sont 
encadrées par des restaurateurs et des diététiciens, et un banquet prestigieux à base de produits oléicoles rassemble les participants dans la salle de la Grande Audience du Palais des Papes.

De cet ensemble se détachent chaque année les expositions commandées par la Chartreuse de Villeneuve-lès-Avignon, qui prolongent pendant quelques semaines l'événement des Oléades, toujours sur la thématique de l'oléiculture. Les années précédentes, ces expositions ont principalement montré des photographies d'art. Une autre fois, des diapositives ont été projetées ainsi que des enluminures évocatrices de l'olivier. A partir de 2002, une partie de l'exposition a été confiée à une association, Artésens, qui regroupe des architectes, des décorateurs, des sculpteurs, des comédiens, et crée sur le thème de l'environnement des parcours sensibles et tactiles particulièrement adaptés aux enfants et aux personnes atteintes de déficiences visuelles. En 2003, cette partie de l'exposition, appelée «L'olivier, balade des sens ", s'est étoffée de nouveaux artéfacts et a été complétée par une exposition de photographies intitulée "L'olivier, arbre de l'unité », appartenant à la chaîne de magasins Oliviers \& Co, spécialisée dans la vente d'huiles du monde entier ${ }^{12}$.

Si la seconde exposition, qui présente dans l'ensemble de la Chartreuse le travail de vingt photographes méditerranéens sur le thème de l'olivier, est relativement conventionnelle avec les clichés des paysages méditerranéens et les textes laudateurs qui les accompagnent ${ }^{13}$, la partie organisée par Artésens nous semble mériter une description plus fine en ce qu'elle révèle l'importance des relations qui existent, autour de l'olivier, entre mise en patrimoine et mise en tourisme. Par exemple, l'exposition «L'olivier, balade des sens. Parcours ludique et tactile » (14 février - 27 avril 2003) s'appuyait sur des manipulations sensorielles et ludiques pour explorer toute la richesse de l'arbre et sa place en Méditerranée. L'exposition était organisée autour de six modules intitulés «La carte puzzle de la Méditerranée », "Paysages de la Méditerranée ", "Les trois religions de la Méditerranée », "Le petit laboratoire », "L'olivier conteur », "La caverne de l'olivier» et elle s'adressait de manière privilégiée à un public touristique, totalement extérieur au monde de l'oléiculture. Le propos de l'exposition était d'

12. Ces expositions sont présentées dans La lettre de la Chartreuse, $n^{\circ} 53$ (janvierjuin 2003).

13. Voir L'olivier, le don de la Méditerranée (1999). 
« apprendre en amusant », en appuyant cet apprentissage sur l'exercice des sens. Les visiteurs étaient invités à suivre les six modules et à participer à l'exposition de manière interactive, successivement en faisant le puzzle, en découvrant des paysages qui présentent les emblèmes touristiques des régions oléicoles dans des boîtes optiques et tactiles, en se livrant à un jeu d'associations d'images concernant les références religieuses de l'olivier, en expérimentant les propriétés physiques des produits oléicoles (densité, émulsion, viscosité, conservation), en écoutant des contes au pied d'un olivier en contreplaqué, en essayant de deviner dans la pénombre des formes sculptées dans un tronc d'olivier.

Avant d'analyser plus en détail ce que mobilise ce type de mise en patrimoine, nous allons présenter une séquence supplémentaire qui est importante pour comprendre la nature des Oléades et en préciser le contexte. Une conférence donnée à la Chartreuse par un dégustateur permet en effet de mesurer l'écart qui existe entre les modes de valorisation patrimoniale que nous avons recensés dans la vallée des Baux et ceux qui sont proposés dans le cadre prestigieux des monuments historiques. Eric Verdier est un spécialiste du goût et de l'analyse sensorielle comparative des produits alimentaires, il a travaillé longtemps pour la chaîne de magasins Oliviers \& Co. La conférence qu'il donne à l'occasion des Oléades sur le thème "Analyse sociale et sensorielle de l'huile d'olive de l'Antiquité à nos jours » est volontiers lyrique. Conçue comme un "hommage à l'arbre suprême ", elle est basée sur l'hypothèse que l'huile d'olive a permis à l'humanité de survivre et de se développer dans un environnement préhistorique dangereux. Le pouvoir astringent de l'olive aurait permis aux hominidés de développer goût et odorat pour analyser les poisons. A travers un ensemble de références hellénistiques et latines qui combinent philosophie et botanique, Eric Verdier veut démontrer que l'huile d'olive fut une base indispensable à la vie. Il explique que chaque olive est différente, à l'image des hommes, et il nourrit son argumentation de considérations chimiques et organiques. Selon lui, nous sommes seulement au début d'une vraie compréhension des produits oléicoles et nous devons aller vers la fabrication de «premiers crus » d'huile d'olive.

Après ce discours qui occulte largement les conditions sociales et culturelles de la production pour ne retenir que des aspects environnementaux, thérapeutiques, gastronomiques, ou métaphysiques, le public composé d'une trentaine de personnes pose des questions de tous ordres qui replacent le sujet dans un cadre concret. Les 
préoccupations du public concernent la taille et les techniques culturales, le goût des huiles, la maturité des olives à la cueillette, les techniques d'extraction de l'huile, les vertus médicinales de l'huile, la résistance à la cuisson, les écarts de prix, la culture intensive, la durée de conservation ou encore les alliances gustatives avec l'ail, le vinaigre ou le vin. Les réponses s'efforcent d'insister sur la spécificité des huiles qui correspondent à des terroirs, sur la nécessité de promouvoir une oléiculture de qualité pour éviter d'entrer en concurrence avec les autres pays producteurs, sur les liens entre la qualité du produit et le soin apporté à l'arbre, sur la simplicité et la grandeur de l'huile, sur la notion de millésime et les progrès à faire pour "tirer la qualité vers le haut ", ou sur les phénomènes d'oxydation de l'huile.

Nous sommes donc ici aux prises avec un ensemble de discours et d'objets qui mettent en valeur l'olivier et les produits oléicoles sur un mode tout à fait spécifique, détaché de l'action des filières professionnelles comme des initiatives qui lient oléiculture et identité locale. Au contraire, l'oléiculture est ici associée à l'universalité des représentations esthétiques, sensorielles et perceptivo-cognitives. Elle est ainsi à même de toucher n'importe qui indépendamment de son origine géographique et a fortiori les personnes qui ne sont là que pour le temps court d'un séjour touristique.

\section{Projets de communication et mise en tourisme des sites culturels}

Des enquêtes complémentaires menées auprès des organisateurs des Oléades montrent que l'événement a été conçu en relation avec l'office du tourisme d'Avignon " pour animer une période creuse ». Le projet articule donc étroitement mise en patrimoine et mise en tourisme. Le thème de l'olivier aurait été choisi en raison de son universalité, de son caractère méditerranéen et du riche contenu qu'il offre aux personnes extérieures à la région. Par rapport aux autres activités de la Chartreuse, les Oléades se donnent pour mission d'animer le monument à l'intention du grand public et des touristes de passage. Elles s'appuient sur l'environnement méditerranéen de la Chartreuse, qui comprend un moulin à huile et une oliveraie, mais n'ont pas de lien direct avec l'autre pôle d'activité de la Chartreuse, soit l'accueil d'artistes en résidence. Conçu par le personnel du centre, l'événement a un lien ambigu avec la filière oléicole qui est ici jugée «frileuse ». Le projet, porté par la Chartreuse, de fait, n'attire pas forcément les producteurs ni les 
professionnels de la région. Cette absence de dynamisme déçoit les organisateurs, mais le sujet plaît et les Oléades sont considérées comme une bonne équation entre le calendrier, le monument, le public local et le public touristique. Au sein des Oléades, les expositions constituent une partie culturelle non prépondérante et les deux mois au cours desquels elles sont présentées s'opposent structurellement aux deux jours de lancement qui attirent un public plus nombreux au Palais des Papes. D'une manière générale, le projet apparaît comme étant ordonné autour des notions de terroir et de qualité d'une part, par la volonté d'animer le patrimoine et de le faire découvrir aux touristes d'autre part. Il s'inscrit ainsi dans une dialectique entre la valeur propre du lieu patrimonial et la valeur ajoutée par l'animation touristique; il propose un événement à contre-courant de la saisonnalité pour opérer un rééquilibrage calendaire des flux.

Le public des expositions a pu être étudié à partir du planning de réservation des groupes. Parmi ceux qui ont visité les expositions des Oléades, les groupes scolaires et les étrangers sont les plus nombreux. Par exemple, plusieurs classes sont venues en bus d'Italie dans le cadre de séjours touristiques scolaires, dans le cadre de programmes thématiques sur le patrimoine; pour les élèves impliqués, le développement de l'offre patrimoniale a entraîné une plus-value qui a justifié l'organisation d'un séjour touristique et culturel. Cette situation a été favorisée localement par l'existence d'une convention entre la Chartreuse et la mairie de Villeneuve-lès-Avignon, prévoyant un tarif préférentiel pour les groupes scolaires. En dehors de ces groupes, quelques associations ont visité les expositions (centres aérés, commissions culturelles municipales, clubs de troisième âge, etc.). Mais dans sa grande majorité, ce public ne semblait pas avoir de liens particuliers avec le monde de l'oléiculture. Pour dépasser ce constat un peu sommaire, nous avons privilégié une approche du dispositif de l'exposition lui-même, à partir de l'observation de ses différents modules. Le fait de visiter plusieurs fois l'exposition et de discuter avec le guide, en particulier, a nourri notre analyse des processus de médiation qui sont à l'œuvre. Sans avoir de formation spécifique concernant la pédagogie ou le travail avec les enfants, ce guide explique que l'exposition s'adresse plutôt à un public "enfantin ou touristique ». Aussi, il conseille aux visiteurs adultes de redevenir momentanément des enfants. Selon lui, ce qui fonctionne le mieux est ce qui calme les enfants ou ce qui éveille leur curiosité en étant insolite. Le guide essaie 
donc de favoriser ces situations en invitant les visiteurs à faire travailler leur imagination par l'intermédiaire des sens et il explique au besoin les modules qui ne sont pas immédiatement compris. En ce qu'elle permet une ouverture vers l'universel, la mise en patrimoine participe ici de la mise en tourisme puisqu'elle s'adresse à tous, sans préjuger de leurs origines culturelles ou géographiques.

Outre ces observations menées sur le site même des expositions, nous avons tiré profit d'un travail antérieur concernant la Chartreuse, qui présente une analyse institutionnelle et s'intéresse au projet de réhabilitation d'une oliveraie sur le site de la Chartreuse. L'analyse institutionnelle révèle qu'il existe une fracture entre le projet culturel du Centre national des écritures du spectacle (résidences d'artistes) et la mise en valeur du patrimoine monumental de la Chartreuse. La distinction est nette aussi bien sur le plan des publics, des budgets, des publications, des manifestations organisées, que sur le plan du personnel. Un espace de conflit potentiel est ainsi créé entre un volet plus culturel et un volet d'animation touristique du site, qui permet de mesurer l'importance d'un style de management « horizontal » (Le Falher 2002 : 89-90).

Selon certains acteurs de la Chartreuse, le projet de réhabilitation de l'oliveraie abandonnée qui est inscrite sur le périmètre classé permettrait dans une certaine mesure de conjurer ces risques de conflit en mettant en œuvre un projet culturel en cohérence avec l'identité du lieu et les impératifs de valorisation touristique du site. Les oliviers, situés dans une zone intermédiaire, permettraient de favoriser un système de renvois entre intérieur et extérieur du site et de constituer un pont entre le patrimoine et son utilisation historique (par les chartreux) ou comme support de création (par les artistes en résidence). Dès lors, le projet a été formalisé et une note interne a prévu les étapes successives de cette opération de valorisation du patrimoine oléicole. Les données topographiques, historiques et institutionnelles concernant le site font apparaître des contraintes liées à la protection qui plaident pour une intervention minimale. Cependant, l'intérêt de l'opération semble évident à plusieurs titres : pour la Chartreuse, la réhabilitation de l'oliveraie vient appuyer la thématique des Oléades et constitue un outil d'intégration au territoire et un facteur de cohérence; pour la municipalité de Villeneuve, le projet s'inscrit dans une politique plus générale d'aménagement des espaces verts; pour le public il constitue un facteur supplémentaire de fréquentation du site. Dès lors, une 
réflexion a été impulsée pour choisir un porteur de projet capable d'obtenir les financements nécessaires.

Nous avons compris ici, à partir de ces approches successives, que la valorisation patrimoniale de l'oléiculture, dès lors qu'elle est mise en œuvre au sein d'une institution culturelle prestigieuse, ne suit pas les mêmes voies qu'à l'accoutumée. L'oléiculture est dans ce cas un moyen beaucoup plus qu'une fin. Les opérations de sa mise en patrimoine mobilisent en conséquence des procédés communicationnels très différents de ceux que nous avions observés à l'échelle de la vallée des Baux. Par leur ambition et leur généralité, ces procédés communicationnels participent alors à la mise en tourisme des sites.

\section{Loléiculture sous le regard d'une institution culturelle}

L'observation des Oléades témoigne finalement d'un mode spécifique de valorisation patrimoniale de l'oléiculture par une institution culturelle prestigieuse, qui ouvre l'espace de l'oléiculture à un public touristique largement extérieur au monde de la production oléicole.

Une première spécificité de la valorisation patrimoniale de l'oléiculture dans le cadre des Oléades est qu'elle se construit en rupture avec le projet culturel global de la Chartreuse et avec le monde des oléiculteurs. La manifestation et les expositions qui l'accompagnent ne sont, aux yeux de leurs concepteurs, qu'une façon parmi d'autres d'animer les monuments historiques dans une période creuse et de rééquilibrer les flux de fréquentation. Ainsi, l'oléiculture est ici un simple prétexte, choisi pour son potentiel pédagogique, ludique et symbolique et pas vraiment pour sa valeur documentaire ou emblématique d'une identité. Le propos est clairement affiché : apprendre en s'amusant. Le discours sur l'oléiculture, largement stéréotypé dans ce cas, n'est qu'une base pour conduire à des expériences sensorielles visant à séduire les touristes parce qu'elles se veulent universelles. Il s'agit là d'un type de requalification bien particulier, qui a trouvé avec l'oléiculture un sujet porteur et qui tente d'en exploiter les potentialités dans une perspective de créativité et de développement touristique. Le discours ambiant sur l'oléiculture, constitué par l'ensemble des opérations de patrimonialisation, sert de toile de fond sur laquelle un public majoritairement touristique est invité à construire du sens et à mieux connaître ses sens. 
Cette situation permet de comprendre la faible implication des oléiculteurs et des acteurs des filières professionnelles dans l'événement. Les seuls professionnels qui interviennent sont des spécialistes de la gastronomie, qui proposent, encore une fois, des médiations d'ordre sensoriel. Le thème de l'oléiculture se libère ainsi de son substrat technique et agricole pour rejoindre d'autres univers de référence. Signe de ce glissement, les expositions, par leur nature comme par les objets qu'elles présentent, ont peu de rapport avec les projets que nous avons évoqués à propos de la vallée des Baux. Ici, le contrat communicationnel n'est pas basé sur la présentation d'un savoir objectif et synthétique concernant l'oléiculture ou les changements qui affectent les pratiques sociales. Dans le cas de l'exposition «L'olivier, ballade des sens ", les objets présentés ne sont ni des outils de travail anciens, ni des panneaux explicatifs, mais des machines interactives construites pour l'occasion et qui permettent au public de participer à l'exposition. Le propos n'est donc pas vraiment d'acquérir des connaissances sur l'oléiculture, mais de mettre en jeu son ingéniosité et ses capacités inventives et imaginatives dans un cadre conçu par des artistes. Dans le cas de l'exposition photographique, de même, les clichés sont présentés d'abord comme des œuvres avant d'être des documents. Leur valeur réside plus dans leur grain ou la lumière qu'ils ont su capter que dans le caractère représentatif de la culture de l'olivier.

Ainsi, les expositions des Oléades s'inscrivent dans l'espace thématique du musée d'art plutôt que dans celui du musée de société. Le rapport au monde d'origine - celui de l'oléiculture - est médiatisé par une recherche de créativité qui s'exprime à plusieurs niveaux : dans l'intégration du projet des Oléades au projet général de la Chartreuse (par exemple à travers la réhabilitation de l'oliveraie comme facteur de cohérence entre projet artistique et environnement), dans la conception et la fabrication des expôts par une association réunissant des artistes et des créateurs, dans l'ambition pédagogique qui invite à la découverte et à l'expérimentation sensorielles, et dans le fait de s'adresser à n'importe qui indépendamment de son ancrage local ou régional. Ici, la valeur n'est pas créée par un simple exhaussement d'objets de «trouvaille» (Davallon 2002 : 76) qui seraient requalifiés en étant simplement extraits de leur contexte, sélectionnés pour leur représentativité et mis au musée, elle relève du travail des artistes qui impose un double filtre à la réalité représentée et modifie ainsi 
considérablement la relation de l'oléiculture à son monde d'origine et la relation du public à l'oléiculture.

Cet état de fait, dans ce type d'exposition, entraîne le régime symbolique de la référenciation à fonctionner sur un mode particulier, à la limite des règles constitutives de l'exposition (Davallon 1999: 3032). En effet, la distinction entre objets exposés et objets qui servent à les présenter n'est pas nette ici. Même si les relations des objets à leur monde de référence sont garanties par un discours plausible, on peut difficilement dire ici que les objets sont authentiques et appartiennent au monde qu'ils représentent. Bien au contraire, ils ont été fabriqués pour l'occasion et sont donc pour la plupart des œuvres. Dès lors, ces expositions s'émancipent de la chaîne des patrimonialisations successives et opposent la création à la conservation. Nous touchons donc avec cet exemple à une conséquence importante de la mise en patrimoine : la requalification sociale et symbolique des objets patrimoniaux est un préalable qui encourage un travail périphérique de mise en valeur artistique de ces mêmes objets par des institutions culturelles spécialisées ${ }^{14}$. Cette mise en valeur artistique et culturelle pourrait bien être l'étape ultime de la patrimonialisation, l'étape où le discours se suffit à lui-même, où le monde d'origine n'existe plus que comme représentation abstraite. Dans ces conditions, les publics idéaux sont les publics qui ne connaissent rien du monde d'origine qui a été mis en patrimoine, c'est-à-dire les publics constitués de personnes extérieures et de touristes. De cette manière, même si elle apparaît souvent comme un facteur secondaire au regard de l'économie des infrastructures ou des équipements touristiques par exemple, la mise en patrimoine aboutit logiquement à la mise en tourisme, car elle construit un rapport d'extériorité avec l'univers de référence des objets patrimonialisés, rapport d'extériorité qui permet aux touristes de s'approprier individuellement un patrimoine régional donné.

14. C'est dans un sens similaire que Dupré (2000: 498) considère les processus de relance et de requalification comme des manières d'ouvrir les objets requalifiés à un sens nouveau : la relance permet alors à ces objets de toucher un public élargi. 


\section{Conséquences théoriques : le tourisme comme agent de requalification patrimoniale}

Les observations qui précèdent s'inscrivent dans le cadre des débats qui ont accompagné en France, depuis une vingtaine d'années, l'émergence du terme de patrimoine et son extension à des données de plus en plus diverses et hétérogènes. Sans aller jusqu'à retracer l'ensemble du processus d'apparition et d'extension du patrimoine, il s'agira ici de cerner les limites des constats critiques réitérés de "pétrification » ou de "muséification », formulés par un certain nombre d'auteurs qui se sont intéressés à la patrimonialisation.

En effet, notre propos consiste à essayer de dépasser ces limites, en vue de comprendre précisément ce que les opérations de mise en valeur du patrimoine engagent face aux processus plus généraux de mise en tourisme. Leurs conséquences, à notre avis, ne se limitent pas à pétrifier une culture vivante autour de quelques signes stéréotypés, mais comprennent au contraire une part active et positive de création de configurations de valeurs nouvelles en vue de toucher des publics nouveaux. Ainsi, notre travail ne s'inscrit pas seulement dans la logique binaire de la dénonciation d'une réification possible de la culture par le patrimoine, mais vise à comprendre par quels processus complexes la culture mise en patrimoine peut faire apparaître un surcroît de valeur, sur un plan économique aussi bien qu'axiologique, et contribuer ainsi au processus de mise en tourisme. Dans ce contexte, nous nous intéresserons d'abord aux théories de la patrimonialisation, puis aux processus de production de valeur et à la distinction qui peut être faite entre valeur économique et valeurs symboliques, avant de focaliser notre attention sur la notion complexe de requalification. L'ensemble de ces données permettra de montrer que, de même que les opérations de mise en patrimoine mettent des ressources locales à la portée des touristes, la mise en tourisme participe d'un processus de requalification patrimoniale qui renforce les potentialités locales de développement économique. 


\section{Les limites de la patrimonialisation}

Dans le contexte des études concernant l'économie touristique et le développement territorial, parler de "mise en patrimoine» ou de "patrimonialisation » a été d'abord un progrès par rapport aux analyses classiques concernant le patrimoine. Ce glissement des problématiques de recherche a suivi le mouvement d'extension du patrimoine qui, centré d'abord autour des monuments historiques et des chefs d'œuvres artistiques, a englobé par la suite des savoir-faire, des traditions populaires, des pratiques sociales, des croyances, des tournures langagières, des éléments de l'environnement, etc.

En même temps que le patrimoine s'est étendu à des réalités immatérielles ou symboliques, il a été de moins en moins définissable en termes de corpus d'objets concrets, il est devenu de plus en plus un objet d'interprétation. Les recherches se sont progressivement concentrées sur les modalités et les ressorts des phénomènes de mise en valeur patrimoniale plutôt que sur la nature des objets hissés au rang de patrimoine, afin de comprendre les mécanismes et les représentations symboliques qui sont à l'œuvre chaque fois que des objets - matériels ou non - sont reconnus comme patrimoine par un collectif. La patrimonialisation, dans cette perspective, renvoie à la fois à l'histoire de la reconnaissance d'éléments culturels comme patrimoine et à la tendance actuelle à mettre en patrimoine un ensemble de plus en plus vaste d'éléments culturels.

Ainsi, la notion de patrimonialisation a permis d'envisager la notion de patrimoine dans une perspective plus dynamique, en permettant d'évoquer les processus de sa mise en valeur plutôt que les objets qu'il vise. Le patrimoine est alors devenu une ressource à part entière pour les communautés qui le mobilisent et a pu être mis progressivement en rapport avec des formes spécifiques de représentation collective de la mémoire et du passé. Pourtant, il semble que la perspective d'une insistance sur les mécanismes de la patrimonialisation arrive à son tour à saturation, comme était arrivée à saturation la vision taxinomique et cumulative classique du patrimoine. En effet, les mécanismes de la patrimonialisation ont été décrits à de nombreuses reprises et peuvent désormais être réduits à quelques lois générales bien connues : la patrimonialisation servirait au développement des territoires, au tourisme, à la gestion culturelle. 
Ce type d'analyse nous semble pourtant laisser de côté une partie de la réalité, en postulant que le patrimoine est un instrument, un outil de développement, mais en refusant de voir en quoi il peut être créatif. Interroger, comme nous le faisons, les conséquences culturelles, sociales ou économiques de la patrimonialisation, c'est donc plaider pour une reconnaissance plus objective et positive des changements induits par la notion de patrimoine sur les représentations que nos contemporains, autochtones ou touristes, se font du monde dans lequel ils vivent.

Dans ces conditions, nous sommes amenés à relativiser les approches critiques des sociologues qui voient dans la mise en patrimoine une façon d'asphyxier la création artistique ou culturelle. Par exemple, les travaux du sociologue Henri-Pierre Jeudy (1999) et ceux qui suivent son point de vue nous semblent devoir être discutés ici. Selon cet auteur, la «muséalisation» des cultures, leur «muséification» revient à une forme de réification. L'exposition patrimoniale est conçue comme une « réduction» qui assimile forcément une culture à un ensemble d'objets (Jeudy 1999: 7 sq.). Plus encore : "L'art et la culture font l'objet d'une véritable entreprise de gestion, fondée sur la séparation des liens entre la vie et la culture » (Jeudy 1999: 7).

La culture serait alors séparée de la vie et entrerait dans l'ordre de l'utilitarisme réflexif, elle ne serait qu'un dispositif moral permettant de protéger les hommes de la nature et de réglementer leurs relations ${ }^{15}$. Mais si ce type d'analyse, qui voit dans le principe de conservation un risque d'aller vers une "culture pétrifiée » (Jeudy 1999: 171), est extrêmement important en ce qu'il permet une approche lucide des fonctions politiques et sociales de l'art contemporain, et si la pensée de Henri-Pierre Jeudy peut stimuler nos propres travaux en insistant sur le caractère éminemment dialectique de tout processus de patrimonialisation de la nature ${ }^{16}$, il nous semble nécessaire d'attirer l'attention sur le fait que nature et culture ne peuvent si uniment être renvoyés dos à dos.

15. Jeudy (1999: 8) reprend cette perspective de Sigmund Freud.

16. A partir de l'exemple du land-art, Jeudy (1999 : 136) va jusqu'à considérer le paysage comme un ready-made utilisable par l'art contemporain. Il cite le philosophe italien Georgio Agamben : "L'art est devenu nature, et la nature est devenue art ", et se réfère à Nietzsche : "Après la mort de l'art, la vie est une œuvre permanente» (Jeudy 1999: 177). 
Ainsi, l'exemple de la mise en valeur patrimoniale des produits naturels - et en particulier de l'oléiculture - nous semble prouver que "l'hystérie collective de l'esthétisation » (Jeudy 1999: 177) est loin d'être le seul motif des opérations de patrimonialisation et que celles-ci génèrent par elles-mêmes des formes sociales nouvelles et dignes d'intérêt. Si la patrimonialisation généralisée pousse l'anthropologie de l'art à être soucieuse quant à l'évaluation et à l'indétermination des formes esthétiques contemporaines, il nous semble qu'elle pourrait au contraire permettre aux anthropologues de comprendre comment l'homme traite la nature à une époque où tout l'en détache. Notre hypothèse est ici que les processus de mise en patrimoine mettent une culture donnée à la portée des touristes, parce qu'en cristallisant les désirs de communautés spécifiques, ils s'efforcent de produire socialement de la valeur et ils incarnent en même temps un certain nombre de valeurs qui ouvrent sur l'universel. En acceptant d'abandonner le point de vue esthétique qui présidait classiquement à la mise en patrimoine, il devient évident que la reconnaissance des produits du terroir au titre de patrimoine ne peut pas relever entièrement d'une logique de "pétrification ". L'intérêt de la recherche consiste alors à définir les effets de la mise en patrimoine et nous pousse à nous interroger sur ce que le patrimoine produit. Ainsi, les opérations de mise en patrimoine ne se limitent pas à pétrifier une culture vivante autour de quelques signes stéréotypés, mais engagent un ensemble complexe d'éléments concernant l'ouverture à l'Autre et la formation de valeur(s). C'est donc ce dernier terme que nous allons interroger dans sa polyvalence.

\section{Valeur(s) du patrimoine : entre logique économique et logique symbolique}

C'est presque sans y songer que nous employons l'expression «mise en valeur du patrimoine ». Est-elle pour autant anodine? Nous ne le croyons pas et nous allons nous efforcer ici de montrer que toute mise en patrimoine est une expérience positive qui peut être comprise à la fois sur un plan économique et sur un plan axiologique. Ainsi, la mise en patrimoine renvoie autant à la valeur économique, qu'aux valeurs morales. C'est cette dimension créative du patrimoine qui lui permet de s'adresser efficacement aux étrangers et aux touristes. Pour mettre cela en évidence, nous avancerons des arguments théoriques de 
plusieurs ordres. Nous verrons d'abord quel type de valeur économique est au fondement du patrimoine et des phénomènes de patrimonialisation. Ensuite, nous traiterons plus largement de la question des valeurs morales créées ou déplacées par la mise en patrimoine, cela sera l'occasion de revenir précisément sur la notion de requalification.

Dans son acception la plus ancienne, la notion de patrimoine renvoie à celle de transmission des biens. Plus récemment, sous la Révolution française, elle fut assimilée à celle de «bien commun ». C'est dire que rien ne la rapproche a priori des notions de conservation ou de sélection sur lesquelles sont fondées un grand nombre de ses analyses critiques actuelles. A maints égards, elle s'éloigne même de ces deux dernières notions, en recouvrant les idées d'usufruit ou de ressource. Le fait de considérer que les biens patrimoniaux peuvent constituer une ressource ou se prêter à une gestion usufruitière, pourtant, n'est apparu que récemment dans les analyses concernant le patrimoine ${ }^{17}$. Ce point de vue revient à insister sur l'usage fait par les groupes sociaux d'un patrimoine et nous renvoie à la distinction classique qu'établissent les économistes entre valeur d'usage et valeur d'échange ${ }^{18}$. Pour comprendre les enjeux de cette réflexion sur le patrimoine en termes d'économie et, de cette façon, comprendre le patrimoine comme une ressource touristique au plein sens du terme, nous présenterons ici des travaux d'anthropologie économique qui tentent d'éclaircir les rapports complexes que peuvent nouer les notions de patrimoine et d'échange. D'une certaine manière, ce type d'approche rejoint les analyses de la muséologie contemporaine, qui nous indiquent que des objets même triviaux et «sans valeur" peuvent prendre une valeur de ressource pour un groupe social donné. Cet apparent paradoxe nous invite à nous interroger sur le fonctionnement spécifique de la notion de valeur.

Par exemple, l'approche que propose Maurice Godelier du fait économique dans L'énigme $d u$ don (1996) est issue des recherches que cet auteur a consacrées depuis une trentaine d'années à l'économie et à la culture matérielle chez les Baruya de Nouvelle-Guinée, ainsi que de

17. Malgré le précédent notable que représente Quatremère de Quincy lorsqu'il estime en 1796 que la conservation, en privant les objets de leur environnement et de leurs usages sociaux habituels, revient de fait à une destruction.

18. Cette distinction, fondatrice chez Karl Marx, avait été introduite au XVIIIe siècle par Adam Smith et a alimenté la plupart des études classiques en sciences économiques. 
ses lectures classiques d'anthropologue. Son hypothèse est que les postulats classiques de l'anthropologie structuralo-fonctionnaliste, selon lesquels la vie sociale est exclusivement fondée sur les échanges, doivent être complétés. Il explique que les sociétés, pour fonctionner, retranchent toujours certaines réalités aux échanges, que ces derniers soient cérémoniels ou marchands. Il discute les théories de Marcel Mauss (1950 [1923-1924]) et de Claude Lévi-Strauss (1950) selon lesquelles l'échange de biens (pour Mauss), de femmes ou de messages (pour LéviStrauss) est au fondement de la société. Il opère une distinction entre les choses qu'on donne, les choses qu'on vend, et les choses qu'on garde mais qu'il ne faut ni donner ni vendre.

En fait, il revisite de manière extrêmement méticuleuse et convaincante le legs intellectuel de Marcel Mauss à partir d'une lecture de son Essai sur le don (1950 [1923-1924]) et du commentaire qu'en fait Claude Lévi-Strauss (1950) et il estime que la lecture lévistraussienne de Marcel Mauss a occulté les réflexions de ce dernier sur l'inaliénabilité de certains éléments culturels. Ainsi, la société est bien fondée sur le don, mais elle accorde aussi de l'importance au fait de garder des richesses et de les soustraire au circuit triangulaire maussien du don (donner - recevoir - rendre). Même dans une économie du don, dit Maurice Godelier, il faut que des objets du même type que ceux qui sont donnés, mais plus beaux, soient exclus des dons (Godelier 1996 : 49-50) ${ }^{19}$. Le social ne se résume donc pas à la somme des formes d'échanges, aux contrats, au symbolique comme Claude Lévi-Strauss semble le croire, mais englobe aussi tout ce que les humains s'imaginent devoir soustraire à l'échange (Godelier 1996 : 52-53). La sphère des échanges et des contrats est ainsi complétée par une sphère où les objets sont transmis et où leur usage n'est pas contractuel mais imaginaire, pour fonder la société sur cette double base. Parmi les objets réputés avoir une âme ${ }^{20}$, il y en a donc certains qui circulent et d'autres qui ne circulent pas: les échanges n'épuisent pas le fonctionnement d'une société (Godelier 1996 : 95-96). Dans ces conditions, il faut être attentif aux différents types de propriété qu'il est possible d'exercer sur les objets,

19. L'auteur renvoie ici à Mauss (1950 [1924] : 224) qui relève chez les Kwakiutl l'existence d'objets de cuivre qui ne sortent pas des familles et dont les objets de cuivre échangés lors des potlatch ne seraient que les satellites.

20. Maurice Godelier fait ici référence à la notion de hau, ou « esprit de la chose donnée » chez les Maoris. C'est cette même notion de hau qui fonde l'analyse des échanges chez Mauss (1950 [1924]). 
de manière à bien distinguer droit de propriété et droit de possession ou d'usage (Godelier 1996 : 126). Les objets précieux et les objets de don « se retrouvent donc entre deux principes, entre l'inaliénabilité des objets sacrés et l'aliénabilité des objets marchands » (Godelier 1996: 132). Ils peuvent être alors compris comme des substituts des objets sacrés d'une part, et donc des dieux, et des objets marchands d'autre part, et donc des personnes.

Cette situation invite à examiner, à côté de ce qui est donné ou échangé, ce qui est gardé et les raisons pour lesquelles ce qui est gardé est sacré. En introduisant l'exemple des objets sacrés Baruya appelés kwaimatnié, sortes de paquets oblongs entourés d'écorce et dont nul ne doit voir ce qui est à l'intérieur (Godelier 1996: 149 sqq.), Maurice Godelier montre que l'imaginaire associé à ces objets transmis est plus important que les relations symboliques qu'ils introduisent. L'imaginaire, ici, précède le symbolique et participe de la construction du réel social et de la production des rapports sociaux de classe et de genre. Plus généralement, les réalités soustraites au don et à l'échange semblent alors constituer le fondement des rapports de pouvoir, ce qui confirme sur notre propre terrain le bien-fondé d'une étude des conséquences de la patrimonialisation, et conduit Maurice Godelier à étudier, à travers l'exemple des sociétés à potlatch, les notions d'objet précieux et de monnaie (Godelier 1996: 202 sqq.). Or ces objets précieux et ces monnaies semblent avoir pour caractère commun de revêtir les attributs des personnes qui les utilisent ou des ancêtres. Ils sont employés pour réactiver un rapport imaginaire et symbolique à l'origine, puisque :

Pour qu'une monnaie circule comme moyen de paiement ou comme richesse, il faut qu'elle soit en quelque sorte autorisée à le faire par ses liens avec une réalité qui, elle, ne circule pas, est tenue hors de la sphère des échanges et se présente comme la source de toute valeur d'échange (Godelier 1996: 226-227).

Ce faisant, ces objets précieux se rapprochent singulièrement de la notion de sacré, qui représente toujours "un certain type de rapport aux origines »(Godelier 1996: 236 sqq.). Dans cette perspective, les hommes réels sont «agis » par des doubles imaginaires qu'ils placent à l'origine d'eux-mêmes; ils ne vivent pas seulement «en société » mais produisent « de la société » pour vivre. Ces hommes imaginaires des origines sont là pour redonner aux hommes leurs propres coutumes sacralisées, transmutées en bien commun (Godelier 1996 : 242-243). Les objets sacrés qui les représentent, en conséquence, sont gorgés de 
sens, ce qui s'oppose à la perspective classique de Claude Lévi-Strauss selon laquelle le sens est plutôt à chercher dans les échanges. Ainsi :

Il ne saurait y avoir de société humaine sans deux domaines, celui des échanges $[\ldots]$ et celui où les individus et les groupes conservent précieusement pour eux-mêmes, puis les transmettent à leurs descendants ou à ceux qui partagent la même foi, des choses, des récits, des noms, des formes de pensée (Godelier 1996 : 281).

Ce sont ces points d'ancrage qui ramènent les individus face à leurs origines et permettent de construire les identités des sociétés et des individus dans le temps. Ces points fixes peuvent être compris comme des dons que les sociétés se font à elles-mêmes.

Ainsi résumée, la pensée de Maurice Godelier s'ouvre sur les notions de sacré et de patrimoine et nous est utile pour appréhender précisément le statut des biens patrimoniaux auxquels nous sommes confrontés sur le terrain. Avec le cas de la mise en patrimoine de l'oléiculture, des objets sont retranchés des échanges marchands et sacralisés : ils permettent de faire entrer en communication autochtones et touristes sur des bases qui ne sont pas uniquement marchandes mais aussi symboliques. Ainsi, à travers l'analyse que fait Maurice Godelier de la formation de la valeur, nous comprenons que sous la dimension économique de la mise en patrimoine est toujours logée une dimension axiologique et mythique, celle-là même qui permet d'intéresser les touristes ou plus généralement d'entrer en communication avec autrui. Son ouvrage est donc déjà un point de départ pour passer d'une problématique de la patrimonialisation à une problématique de la requalification.

Enfin, à ce stade de l'exposé, il convient de rapprocher les analyses de Maurice Godelier des pensées des sémiologues (Davallon 2002) sur la fabrication du patrimoine. Pour qu'il y ait patrimonialisation, six étapes seraient nécessaires : la découverte de l'objet comme trouvaille, la certification de l'origine de l'objet, l'établissement de l'existence du monde d'origine, la représentation du monde d'origine par l'objet, la célébration de la trouvaille de l'objet par son exposition et l'obligation de transmettre aux générations futures. La patrimonialisation, à ce compte, est bien une manière de produire de la croyance en renvoyant les objets à une origine imaginée et en les donnant à voir aux personnes venues de l'extérieur. Transposé aux sociétés touristiques, le processus 
consiste alors à extraire les objets patrimoniaux de leur contexte d'origine pour les donner à consommer aux touristes.

\section{Nature sociale de la valeur produite par le patrimoine}

Mais alors, quelle est la nature de la valeur produite par le patrimoine? Si de la valeur peut être socialement produite à partir de la culture, il ne semble pas qu'elle soit uniquement d'ordre économique. Avant de compléter ce qui a été dit par une approche plus large des valeurs au pluriel, nous allons faire référence ici à un autre travail d'anthropologie économique qui s'est efforcé lui aussi de saisir dialectiquement la question des échanges marchands. Il s'agit de la thèse que Michèle de La Pradelle (1996) a consacrée au marché forain, au marché de la truffe et au marché-gare de Carpentras (Vaucluse).

Le grand mérite de ce travail, selon nous, est d'éviter de renvoyer dos à dos ce qui relèverait de l'économique d'une part, du culturel d'autre part. Le but poursuivi par l'auteure est de vaincre le préjugé selon lequel là où les enjeux économiques sont importants, les rapports sociaux se diluent et inversement. Il s'agit ainsi de lutter contre un certain "économisme », qui voudrait que la fonction actuelle des marchés ne soit plus économique, mais liée à des impératifs d'animation et au besoin de fournir un "supplément d'âme » dans une société par ailleurs résolument marchande. Cet « économisme » empêcherait de :

Considérer que l'échange marchand est lui-même en tant que tel un rapport social [où] dans un laps de temps donné [...] les acteurs se reconnaissent simplement comme des partenaires équivalents, si inégaux que soient par ailleurs leur pouvoir ou leur statut (La Pradelle $1996: 15)$.

Cette situation entraîne dans les marchés la mise en place d'un espace social spécifique qui permet de définir l'échange marchand comme un rapport social inscrit dans la modernité mais situé en marge de la vie sociale ordinaire et des inégalités qui la régissent. Les marchés, en somme, mettent en scène selon des modalités variables (rituelles ou spectaculaires, ludiques ou sérieuses) un même principe d'égalité.

Ce dernier exemple donne un coup fatal à l'opposition artificielle qui est trop souvent postulée entre économie et culture et nous conduit à relativiser la distinction que nous avions faite dans un premier temps entre valeur et valeurs. Les rapports dialectiques qui se nouent entre 
patrimoine et échange révèlent que les rapports entre patrimoine et valeur ne se réduisent jamais à des questions purement économiques. Le patrimoine, en effet, ne se limite pas à créer de la valeur, il semble surtout produire des valeurs ou déplacer ces dernières. Notre approche des conséquences de la patrimonialisation sur le tourisme, dès lors, interroge un ensemble de requalifications symboliques provoquées par la mise en patrimoine. A cette condition, les changements de statut des objets patrimonialisés peuvent être reconnus comme des changements d'ordre axiologique plutôt qu'économique. La patrimonialisation des produits oléicoles crée du lien social, des occasions de rencontre et d'échange avec des touristes ou tout simplement avec des personnes venues de l'extérieur, tout autant qu'une plus-value économique.

\section{Requalification spatiale et hauts lieux touristiques}

Pour mieux comprendre ce qui se passe lorsqu'un patrimoine est inventé comme ressource touristique, nous allons désormais aborder la question des valeurs créées ou déplacées par la mise en patrimoine, en nous appuyant principalement sur la notion de requalification. Cette notion, héritée de l'urbanisme et de l'architecture, comprend un processus de production de lien social et de culture qui intéresse le tourisme. Dès lors, le propos de notre travail n'est pas tellement de constater une fois de plus la réalité et le devenir de la patrimonialisation, mais plutôt d'insister sur la manière singulière par laquelle la reconnaissance d'un ensemble de réalités comme patrimoine induit un changement d'univers de références et une requalification des contenus considérés, ce qui permet d'intéresser des personnes extérieures à la culture du territoire considéré.

Pour envisager d'abord ce qui relève sur notre terrain d'une requalification spatiale, nous allons essayer de transposer le concept de " haut lieu» tel qu'il a été avancé dans des textes réunis par André Micoud dans l'ouvrage Des hauts lieux. La construction sociale de l'exemplarité (1991). Les « hauts lieux» ont fait l'objet de nombreux débats et notamment d'un séminaire au ministère de l'Equipement en 1989. Ils s'inscrivent donc dans un ensemble de réflexions qui n'est ni passéiste, ni historique, mais qui s'efforce au contraire de comprendre comment les sociétés appréhendent le changement et la tradition en manipulant symboliquement leur espace. C'est la production symbolique 
des lieux exemplaires qui est placée ici au centre de la réflexion, et l'enjeu est d'étudier comment les lieux qui veulent signifier quelque chose de grand ou d'essentiel ont été choisis, transformés, ou au contraire oubliés.

Ces lieux sont des lieux « exemplaires » qui ont valeur de paradigme, de spécimen, et de modèle inimitable. Ainsi, ils laissent penser que d'autres espaces sociaux sont possibles et ouvrent une dimension utopique dans laquelle leur production signifie la possibilité d'un avenir différent. Leur création suit des logiques spécifiques qui renvoient à la gestion de la mémoire collective et sont appuyées sur des collectivités initiatrices selon une périodisation spécifique. Dans ce cadre, l'analyse des lieux du pouvoir et de leur construction est particulièrement éclairante.

L'étude de cas concrets permet de reconnaître le critère de fréquentation touristique comme la cause ou comme l'effet de la production d'un haut lieu. Pour qu'un site touristique soit reconnu comme haut lieu, Jean Davallon (1991) expose les conditions qui doivent être réunies. Le lieu doit d'abord être porteur de sens, soit parce que son interprétation apporte un savoir sur son usage passé, soit pour sa valeur esthétique, soit pour sa signification transcendante. La mise en valeur du lieu passe ensuite par une mise en exposition qui est de l'ordre du rituel : la logique de l'exemplarité est présentée au visiteur par une double coupure qui forme système, entre passé et présent, entre ici et ailleurs. Le haut lieu fonctionne alors comme un indice, qui permet le contact et en même temps renvoie à un ordre de réalités plus général. La production d'un haut lieu, ainsi, utilise au mieux le capital symbolique d'un site en vue de créer un accord entre site naturel, histoire et objets. Pour ce faire, les opérations de production des hauts lieux portent sur plusieurs plans : elles jouent sur la façon dont le site apparaît, elles présentent un savoir de manière plus ou moins discrète, elles règlent les pratiques de visite, elles prévoient des stratégies qui permettront aux visiteurs de comprendre le site ou le monument comme un indice. Ainsi, elles suivent la logique de la production du patrimoine, basée sur une triple opération de mise en communication, de mise en exposition et de mise en exploitation. En fin de compte, la production des hauts lieux est définie par le paradoxe qui existe « entre une pratique de production répondant à un modèle économique gestionnaire et une pratique de réception répondant à un modèle symbolique d'adhésion à une œuvre » (Davallon 1991: 102). 
Les hauts lieux nous fournissent un modèle heuristique intéressant pour penser la mise en valeur du patrimoine rural et ses conséquences sur le tourisme. Ils nous montrent qu'un espace quelconque, à condition d'être convenablement " produit » dans le cadre d'un dispositif collectif approprié, peut être requalifié et prétendre à une valeur « exemplaire » d'indice d'une réalité plus générale. Dans le cadre de tels processus, l'exemple du paysage oléicole mérite d'être développé. Une oliveraie abandonnée, pour peu qu'elle s'intègre à un site reconnu, vaudra d'être réhabilitée et cette opération suscitera débats et discours ${ }^{21}$.

Mais les notions de « haut lieu » et de "paysage », quoique basées sur la matérialité des territoires, évoquent déjà les dimensions du social et du symbolique, dans la mesure où le paysage est un «donné construit par une perception, elle-même informée par des schèmes conceptuels » (Gérard Lenclud, cité par Dubost et Lizet 1995 : 227). Autrement dit, le paysage englobe à la fois une réalité, l'image de cette réalité et les références culturelles à partir desquelles cette image se forme (Dubost et Lizet 1995). Ainsi, pour comprendre les liens qui unissent mise en patrimoine et mise en tourisme, faut-il désormais prendre en compte les dimensions sociales et symboliques des processus de requalification que nous nous proposons d'étudier.

\section{Production du patrimoine et requalification sociale et symbolique}

Pour ce faire, nous allons ici transposer à notre terrain des protocoles de recherche qui ont été mis en œuvre par les sciences humaines depuis quelques années à propos des objets matériels ou à propos de certains produits du terroir (Bromberger et Chevallier 1999; Rautenberg, Micoud, Bérard et Marchenay 2000). Si la question de la requalification sociale et symbolique des objets techniques est ici importante, en interrogeant par exemple le statut des objets agricoles liés à l'oléiculture et récemment patrimonialisés, il faut être attentif aussi à la question des procédures de labellisation, et tout particulièrement celle d'Appellation d'origine contrôlée, qui pose des questions relatives à la patrimonialisation du vivant. Sur un plan plus général, ces données supplémentaires nous permettront d'insister sur les différentes

21. Voir supra notre étude de cas de la réhabilitation d'une oliveraie à Villeneuvelès-Avignon. Voir aussi, sur le cas d'une oliveraie réhabilitée à Manosque (Sadorge, Chevallier et Morvan $1996: 16$ ). 
conceptions qui s'affrontent dans le champ du patrimoine rural et sur les meilleures manières de les rassembler.

La perspective développée par Christian Bromberger et Denis Chevallier (1999) ouvre une première voie d'accès aux requalifications sociales et symboliques des objets et des activités techniques. Ils montrent comment les objets techniques s'inscrivent dans des réseaux qui englobent leur production et les échanges dont ils sont le support. Plusieurs exemples mettent en scène des objets bricolés ou adaptés, dans le champ de l'artisanat, de l'agriculture ou de l'industrie. Les circuits que parcourent les objets les voient changer de fonction et investir des espaces sociaux et symboliques nouveaux. Au cours de leurs " carrières ", les objets techniques sont réinventés par leurs utilisateurs. Lorsqu'ils sont présentés comme authentiques, cela n'empêche jamais que des mutations ou des innovations se manifestent. Cette approche permet de dépasser les postulats théoriques classiques de la technologie qui se concentraient soit sur l'articulation entre systèmes techniques et organisation sociale et symbolique, soit sur l'étude des chaînes opératoires, des matières, des outils, des gestes et des processus de production technique. Ici, l'analyse vise les modalités d'appropriation, de domestication, d'interprétation des artéfacts standardisés et s'intéresse aux usages, aux adaptations, aux bricolages. Il s'agit de rendre compte de la vie sociale d'objets qui « rendent visibles les catégories de la culture » (Mary Douglas, citée par Bromberger et Chevallier 1999). Dès lors, l'étude des relances, des revitalisations et des requalifications prend une importance accrue, pour analyser la reproduction d'objets emblématiques selon des techniques singulières et observer ainsi les nouveaux rapports qui apparaissent entre tradition et innovation technique en contexte touristique. Ainsi, chaque fois que nous avons affaire à des objets techniques liés à l'oléiculture et pris en charge par des opérations de mise en valeur du patrimoine, il est utile de nous référer à ce type d'approche. Mais cette perspective est insuffisante pour l'analyse des conséquences sociales, économiques et culturelles de la patrimonialisation de l'oléiculture. En particulier, elle nous oblige à diviser notre objet de recherche à partir du schème général de la production. Pour éviter cet écueil, il nous semble possible d'appréhender les produits oléicoles dans leur ensemble, en faisant référence à un autre travail collectif.

Les recherches rassemblées par Michel Rautenberg, André Micoud, Laurence Bérard et Philippe Marchenay dans l'ouvrage Campagnes de 
tous nos désirs. Patrimoines et nouveaux usages sociaux (2000) permettent en effet d'aller plus loin dans notre réflexion et d'englober, en plus des objets techniques propres au secteur considéré, l'ensemble des réalités liées aux phénomènes de patrimonialisation du vivant. Cet ouvrage et les débats qu'il a suscités et qu'il suscite encore permettent de mieux comprendre la nature des processus de requalification engagés par les différentes opérations de mise en patrimoine.

Dans l'avant-propos de cet ouvrage, Denis Chevallier montre que la valorisation et la marchandisation du patrimoine sont devenues des leviers essentiels pour l'aménagement et le développement des territoires (Sadorge, Chevallier et Morvan 1996). Cette nouvelle «économie du patrimoine », qui implique les chercheurs dans les domaines de l'aménagement et du développement, invite selon lui à une recherche "pluridisciplinaire, ouverte sur l'horizon européen, soucieuse de ses applications » (Chevallier 2000 : xiii) dont l'enjeu principal serait de pouvoir "définir l'objet patrimoine à travers les catégories élaborées par les acteurs eux-mêmes » (Chevallier 2000 : x). L'objectif général du travail est alors d'opérer une "lecture critique des pratiques des institutions confrontées à cet engouement de nos contemporains pour le patrimoine » (Chevallier 2000 : xi). Et plus généralement de «mettre à jour les processus par lesquels une société comme la nôtre recycle en permanence ses productions symboliques dans de nouveaux objets et de nouvelles valeurs »(Chevallier 2000 : xiv).

Parce que le patrimoine est devenu désormais dans nos campagnes un objet culturel, un produit de consommation et un élément du cadre de vie, les auteurs estiment d'abord qu'il devrait entraîner une réévaluation des notions de nature, de sauvage ou de territoire. Le but est alors de comprendre comment les campagnes construisent la patrimonialisation. Dans cette perspective, les thèmes du territoire, des produits, ou de la relation à l'urbain semblent pouvoir insister sur les nouvelles formes de dialogues qui se créent entre acteurs et institutions, sur l'articulation entre biens économiques et valeurs culturelles, sur les manières dont tiennent ensemble des espaces et des groupes sociaux hétérogènes et sur les implications des nombreux acteurs sociaux engagés dans la patrimonialisation (Rautenberg, Micoud, Bérard et Marchenay $2000: 1$-2). Une première distinction essentielle apparaît alors entre patrimoine alimentaire et patrimoine bâti, entre des produits du terroir qui voyagent et des fermes rurales beaucoup plus liées à leur terroir. 
Dès lors, les auteurs proposent de suivre trois directions complémentaires pour analyser la patrimonialisation. Une première voie est tracée par la question des territoires et des enjeux politiques liés à leur définition. Un deuxième mode d'analyse vise à recentrer la réflexion sur l'exemple des produits du terroir, qui se situent à mi-chemin entre le culturel et l'économique. Une troisième voie de réflexion traite de la recomposition des rapports entre ville et campagne et sur la fin de leur opposition. La proximité de ces travaux et de la question des conséquences de la patrimonialisation de l'oléiculture est aisément perceptible. Les actions de requalifier, d'instituer, de nommer, de revitaliser, et le fait qu'elles concernent des produits du terroir, apparaissent en particulier importants ${ }^{22}$.

Avant de montrer comment la notion de requalification se prolonge aussi dans le registre du sensoriel, nous voudrions envisager les enjeux de l'approche que nous venons de présenter, en renvoyant à un débat récent qui a mis en cause le point de vue adopté par les auteurs de l'ouvrage Campagnes de tous nos désirs. Patrimoines et nouveaux usages sociaux (2000). Ce débat nous éclairera sur les limites de la perspective théorique adoptée ici.

Dans un article récent, les sociologues Gilles Laferté et Nicolas Renahy (2003a) estiment que l'ouvrage concerné, inspiré d'une analyse constructiviste de la tradition, développe une analyse constructiviste du patrimoine rural qui contribue à elle seule à produire du patrimoine rural. La perspective développée par Michel Rautenberg, André Micoud, Philippe Bérard, Laurence Marchenay et leurs collaborateurs relèverait alors d'un «volontarisme scientifique de construction patrimoniale » (Laferté et Renahy 2003a : 227) et risquerait de donner à croire à une capacité d'auto-patrimonialisation des lieux.

En réponse à cette analyse, Michel Rautenberg, André Micoud, Philippe Bérard et Laurence Marchenay (2003) expliquent que les conditions de production de leur recherche sont suffisamment mises à jour par l'ouvrage. Le débat introduit par Gilles Laferté et Nicolas Renahy (2003a) équivaut selon eux à questionner la légitimité de leur parole. Ils rappellent que l'analyse du patrimoine, fût-elle

22. Dans cette perspective, il serait intéressant de comparer le dossier de l'oléiculture avec ce qui a été dit d'autres produits valorisés socialement et symboliquement, comme le champagne (Brochot 2000) ou le foie gras (Coquart et Pilleboue 2000). 
constructiviste, n'est pas la même chose que la production du patrimoine, et que la neutralité est impossible dans la mesure où le patrimoine relève toujours des politiques publiques qui l'instrumentalisent. En réponse à cette réponse, et pour clore le débat, Gilles Laferté et Nicolas Renahy (2003b) définissent le patrimoine comme un langage commun créé par la reconfiguration dynamique des mondes scientifique, politique et économique. Selon eux, la politique nationale institutionnalisée, dont relèvent les appels d'offres de la Mission du patrimoine ethnologique, fait le lien entre les différents articles de l'ouvrage coordonné par Michel Rautenberg, André Micoud, Philippe Bérard et Laurence Marchenay (2000).

De cette controverse, il ressort d'abord que nous devons être attentifs au contexte de la recherche comme à celui des acteurs. Ainsi, dans les questions patrimoniales, l'ethnologie apparaît autant comme un point de vue que comme une discipline. Les conclusions que ce débat convoque restent révélatrices d'une situation de concurrence dans la définition du patrimoine et des valeurs qu'il recouvre. Des arguments économiques, politiques, scientifiques, sociaux, culturels, esthétiques, concourent à la définition du patrimoine et à sa valorisation. Ces discours permettent de requalifier spatialement, socialement et symboliquement des éléments culturels, de reconnaître à ces derniers une valeur nouvelle. Mais ces discours seraient-ils suivis d'effets si les processus de requalification ainsi définis ne s'appuyaient pas sur l'expérience empirique des personnes qui les prennent en charge ? Puisqu'il est question ici de produits du terroir, de tourisme et d'ouverture à l'Autre, nous allons considérer désormais les manières dont la requalification et la transformation du sens s'appuient sur le registre sensoriel.

\section{Requalification sensorielle et mise en tourisme}

Nous avons vu comment des éléments culturels peuvent être requalifiés et revalorisés sur des bases spatiales, sociales ou symboliques, ce qui permet de les reconnaître comme patrimoine et d'en faire des réceptacles d'une valeur que la société est susceptible de déplacer en fonction de ses goûts, en particulier lorsqu'elle est confrontée aux goûts des touristes. L'exemple des produits oléicoles, que nous avons choisi d'étudier, nous invite à considérer aussi bien le sens métaphorique que le sens réel du terme de goût, afin de voir précisément comment les processus de requalification liés à la patrimonialisation sont légitimés 
par leur inscription dans le registre sensoriel. Nous ferons référence ici à un exemple de recherche menée sur ces questions, puis nous le mettrons en perspective de manière plus générale.

Dans un article consacré à «La nouvelle culture du vin ", l'anthropologue Jean-Pierre Albert (1989) montre comment peut être légitimée une expérience esthétique du vin, fondée notamment sur le rituel de la dégustation. Avec l'exemple de l'exposition « La vigne et le vin », présentée en 1989 dans le cadre moderniste de la Cité des Sciences et Techniques de La Villette, il tente d'interroger le présent d'une culture du vin, malgré la consonance souvent traditionnelle du produit. Cette culture serait déterminée à la fois par une recherche d'enracinement et de traditionalité, par les innovations liées à l'entrée des chimistes dans les caves et par l'évolution des goûts et des modes. Notant l'entrée massive du vin dans notre culture, Jean-Pierre Albert insiste sur les stratégies de légitimation qui lui sont associées. La pratique de la dégustation nourrit son questionnement :

Le sens du goût, dont le langage (et aussi la philosophie) avait fait le symbole d'un discernement esthétique incapable de définir ses principes, serait-il donc aujourd'hui doté de la plus grande objectivité ? (Albert 1989: 120).

Ici, l'esthète cautionne le chimiste et le chimiste cautionne l'esthète pour aller vers une définition quantitative des qualités et des défauts du vin. Mais si la dégustation est un art,

lequel des Beaux-Arts pourrait-il se vanter d'une maîtrise technique aussi poussée des relations entre paramètres objectifs et effets sur la subjectivité ? (Albert 1989: 120).

En fait, l'objectivation du goût dont témoigne l'expérience de la dégustation est basée sur un ensemble de formules de légitimation qui sont autant de figures de transfert et qui mettent en place un jeu social autour du vin: le livre devient livre d'art quand il parle du vin, des expositions associent vin et architectures d'exception, des étiquettes de bouteilles de vin sont réalisées par des peintres célèbres, etc. Dans ce jeu social qui se met en place, la dégustation est une pratique qui relève autant de l'hédonisme que de la technique ou du snobisme, et les notions de goût physiologique et de bon goût sont étroitement intriquées. La compétence du dégustateur, ainsi, est basée non seulement sur le fait d'apprécier et de connaître le vin, mais aussi sur le fait de savoir en parler et d'enrichir son vocabulaire. Le vocabulaire de la 
dégustation mêle donc évaluation objective et valorisation, et pose la question des limites d'une objectivation des catégories esthétiques. En conséquence, l'expérience esthétique du vin comprend une large part de discours et de valorisation sociale, ce qui incite à relativiser le plaisir des sens dans l'expérience esthétique.

Cet exemple, semble-t-il, permet de comprendre un peu mieux comment s'articulent expérience individuelle et exigences collectives dans l'élaboration d'un produit ou d'un goût. La requalification sensorielle des produits passe par l'élaboration d'une langue spécifique, d'une esthétique et d'un ensemble de discours de valorisation, qui viennent appuyer des savoir-faire et des qualifications spécifiques (goûter, sentir). Dans ce processus, une culture est fabriquée à partir d'une expérience partagée qui est basée sur les actions de qualifier, d'instituer et de nommer. Cela semble correspondre aux caractères généraux de toute expérience sensorielle : sentir, percevoir, nommer, mémoriser, catégoriser, transmettre (Candau 2000 : 6). Ces actions sont fondées sur des transferts de sens et imposent des changements d'univers de référence aux produits concernés. Elles imposent notamment la création d'un langage adapté aux valeurs créées par la nouvelle culture ${ }^{23}$. Pour le cas des produits oléicoles, la requalification symbolique passe par le décalque d'un modèle œnologique. La mise en patrimoine induit bien le basculement d'un univers de référence à un autre. Les produits oléicoles passent d'un registre culinaire à un registre gastronomique, en même temps que la langue de l'œnologie les contamine et en fait des réalités de plus en plus complexes et de plus en plus "sensibles " 24 . La recherche porte alors sur la manière dont les sens viennent au secours du sens pour aider à la production des valeurs.

L'exemple du vin est facilement transposable aux produits oléicoles. Dans le cas de l'apprentissage de la dégustation de l'huile, les descripteurs appris par les sujets sont des acquisitions qui nécessitent l'élaboration d'un vocabulaire spécifique partagé par un certain nombre d'individus. Les processus cognitifs qui mettent en place ce vocabulaire permettent au dégustateur d'objectiver sa perception grâce à des mots que d'autres dégustateurs lui ont appris et/ou avec qui il les partage. Le souvenir et

23. Voir les exemples de classification des odeurs donnés par Candau (2000 : 143 147).

24. Dénommer une réalité linguistiquement, c'est l'identifier. La langue peut donc être considérée comme le premier opérateur patrimonial. 
l'analyse des goûts sont étroitement dépendants de ce processus. Ainsi, sans vocabulaire et sans concertation avec autrui le sujet ne peut progresser dans l'identification des goûts et dans leur classement (arbitraire et social). D'autre part, le dégustateur établit un lien entre les goûts de l'huile et les activités de l'oléiculteur. Ce lien permet d'identifier et de classer mentalement les saveurs, par exemple en désignant comme «fruité noir » le goût d'une huile élaborée avec des olives cueillies tardivement et stockées quelques temps avant la pression, et comme «fruité vert » le goût d'une huile faite avec des olives cueillies tôt dans la saison et rapidement pressées.

En partant de l'expression "mise en valeur du patrimoine ", nous avons donc essayé de montrer que toute mise en patrimoine est une expérience positive qui engage sur un plan économique la création de valeur et sur un plan axiologique celle de valeurs. Pour ce faire, nous avons vu d'abord quel type de valeur est au fondement du patrimoine et quelle forme de valeur est créée par les phénomènes de patrimonialisation. Ensuite, nous nous sommes concentrés sur la question des valeurs créées ou déplacées par la mise en patrimoine, à partir de la notion de requalification que nous avons déclinée suivant les registres du spatial, de social, du symbolique et du sensoriel. Nos résultats tendent à prouver que la patrimonialisation est une forme d'institution de la culture basée sur la figure du transfert, sur un changement d'univers de référence des objets considérés et sur une valorisation de type linguistique. C'est ce transfert qui devient efficace dans un contexte de changement social et culturel, lorsque les regards extérieurs des touristes entraînent les références locales à se recomposer.

\section{Conclusion}

Les pages qui précèdent, tant du point de vue des faits étudiés que des analyses produites, donnent des éléments pour comprendre ce qui se advient dans des sociétés où les interactions avec les touristes vont en s'intensifiant. En Provence, les éléments emblématiques du patrimoine ethnologique régional que constituent les produits oléicoles sont progressivement transformés en ressources culturelles et donnés à consommer aux regards touristiques. Coupés de leur univers de référence habituel, ces éléments sont exhaussés en patrimoine par les agents du patrimoine comme par les promoteurs du tourisme; ils changent de sens pour toucher un public touristique extérieur indépendamment de 
ses origines géographiques et culturelles. A la première étape de la patrimonialisation, qui consiste en une prise de conscience locale et en une tentative de sauvegarde des éléments jugés menacés, succèdent les phases de la critique, durant laquelle les objets valorisés dans la phase précédente sont jugés stéréotypés, puis une phase ultime où le caractère patrimonial des objets est recherché en dehors de leur monde d'origine.

Les deux premières phases de reconstruction ont permis d'élargir l'univers de référence, au point que la patrimonialisation peut être basée désormais sur la notion de transfert. Dans le cas de l'oléiculture, cette phase apparaît par exemple lorsque des comparaisons sont établies entre le monde de l'olivier et celui du vin. Ces connexions nouvelles déplacent la patrimonialisation des objets eux-mêmes vers leur appréciation esthétique, selon un registre plus général et propre à être compris par les personnes extérieures et notamment par les touristes. Ainsi, à la Chartreuse de Villeneuve-lès-Avignon, l'olive devient un simple prétexte pour parler du goût ou des sens en général. Les références au monde d'origine de l'oléiculture sont médiatisées par un système de références présenté par des machines ludiques et interactives dans une ambition pédagogique. Dès lors, le type de référenciation change et il n'y a plus de lien explicite entre les objets présentés dans le dispositif de patrimonialisation et le monde de l'oléiculture. L'objet patrimonialisé entre dans un nouveau système de référence qui le rend disponible auprès d'un public élargi.

Ainsi, l'ouverture d'un horizon culturel donné au tourisme s'accompagne inévitablement d'une requalification des éléments préexistants de la culture locale. Ces éléments prennent des sens de plus en plus variés, tout en suivant des modes de valorisation unifiés mise en fête et mise en exposition, principalement - pour toucher le plus grand nombre. Le processus de requalification ainsi décrit éclaire le phénomène de la mise en tourisme en ce qu'il conjugue mise en patrimoine et mise en tourisme ; il transforme certains éléments clés du patrimoine régional en ressources touristiques, permet aux touristes de se les approprier, et accompagne ainsi, sur le plan des représentations, les transformations des infrastructures, des équipements et de l'offre touristique en général. 


\section{Références}

Albert, Jean-Pierre. 1989. «La nouvelle culture du vin». Terrain, 13 : $117-124$.

Brochot, Aline. 2000. «Champagne : objet de culte, objet de lutte». In Michel Rautenberg, André Micoud, Laurence Bérard et Philippe Marchenay (dir.). Campagnes de tous nos désirs. Patrimoines et nouveaux usages sociaux: 75-90. Paris: Editions de la Maison des sciences de l'homme.

Bromberger, Christian et Denis Chevallier (dir.). 1999. Carrières d'objets. Paris : Editions de la Maison des sciences de l'homme.

Candau, Joël. 2000. Mémoire et expériences olfactives. Anthropologie d'un savoir-faire sensoriel. Paris : PUF.

Chevallier, Denis. 2000. «Avant-propos». In Michel Rautenberg, André Micoud, Laurence Bérard et Philippe Marchenay (dir.). Campagnes de tous nos désirs. Patrimoines et nouveaux usages sociaux: ix-xv. Paris : Editions de la Maison des sciences de l'homme.

Coquart, Dominique et Jean Pilleboue. 2000. «Le foie gras : un patrimoine régional ?». In Michel Rautenberg, André Micoud, Laurence Bérard et Philippe Marchenay (dir.). Campagnes de tous nos désirs. Patrimoines et nouveaux usages sociaux: 91-104. Paris : Editions de la Maison des sciences de l'homme.

Davallon, Jean. 1991. "Produire les hauts lieux du patrimoine ». In André Micoud (dir.). Des hauts lieux. La construction sociale de l'exemplarité : 85-102. Paris: Editions du CNRS.

—. 1999. L'exposition à l'œeuvre. Stratégies de communication et médiation symbolique. Paris : L'Harmattan.

—_. 2002. "Comment se fabrique le patrimoine?». Sciences Humaines, $36: 74-77$.

Dubost, Françoise et Bernadette Lizetm. 1995. "Pour une approche ethnologique du paysage ». In Claudie Voisenat (dir.). Paysage au pluriel: 225-240. Paris : Editions de la Maison des sciences de l'homme.

Dupré, Lucie. 2000. Du marron à la châtaigne d'Ardèche. La relance d'une production agricole "traditionnelle ». Thèse d'ethnologie, Université Aix-Marseille I.

Fournier, Laurent Sébastien. 2005. La fête en héritage. Aix-en-Provence : Publications de l'Université de Provence.

Godelier, Maurice. 1996. L'énigme du don. Paris : Fayard.

Jeudy, Henri-Pierre. 1999. Les usages sociaux de l'art. Strasbourg : Editions Circé. 
L'huile d'olive : de la culture traditionnelle au symbole alimentaire mondialisé. 2002. Paris: Musée national des Arts et Traditions populaires / Campagne d'acquisition R.M.N. (Rapport d'enquêtes-collectes 2002 et proposition de renouvellement 2003. Document de travail interne, non publié).

L'olivier, le don de la Méditerranée, 1999. Introduction par Olivier Baussan. Paris : Editions du Bottin Gourmand / Oliviers \& Co.

Laferté, Gilles et Nicolas Renahy. 2003a. "Campagnes de tous nos désirs... d'ethnologues ». L'Homme, 166 : 225-234.

—. 2003b. "L'ethnologue face aux usages sociaux de l'ethnologie ». L'Homme, $166: 239-240$.

La lettre de la Chartreuse 53. 2003. Villeneuve-lès-Avignon : Centre national des écritures du spectacle.

La Pradelle, Michèle de. 1996. Les vendredis de Carpentras. Faire son marché en Provence ou ailleurs. Paris : Fayard.

Le Falher, Olivier. 2002. Pour une approche des résidences d'artistes comme forme énonciative. Mémoire de DESS : Stratégies du développement culturel, Université d'Avignon.

Les Oléades. 2001. Bulletin semestriel des Etudes vauclusiennes. (Actes du colloque de l'an 2000). Avignon-Villeneuve : Editions RMG / Palais des Papes.

Lévi-Strauss, Claude. 1950. «Introduction à l'œuvre de Marcel Mauss ». In Marcel Mauss (dir.), Sociologie et anthropologie: vii-lii. Paris : PUF.

Mauss, Marcel. 1950 [1923-1924]. «Essai sur le don. Forme et raison de l'échange dans les sociétés archaïques ». In Marcel Mauss (dir.), Sociologie et anthropologie: 143-279. Paris : PUF.

Micoud, André (dir.). 1991. Des hauts lieux. La construction sociale de l'exemplarité. Paris: Editions du CNRS.

Rautenberg, Michel, André Micoud, Laurence Bérard et Philippe Marchenay (dir.). 2000. Campagnes de tous nos désirs. Patrimoines et nouveaux usages sociaux. Paris : Editions de la Maison des sciences de l'homme.

- 2003. «Et si nous prenions nos désirs en compte?». L'Homme, $166: 235-238$.

Sadorge, Jean-Luc, Denis Chevallier et Guy Morvan (dir.). 1996. Quand le patrimoine fait viure les territoires. Paris : Editions du CNFPT. 